\title{
Acne Flares among University Female Students: The Role of Perceived Factors
}

\author{
Amal A. Kokandi \\ Rabegh College of Medicine, King Abdulaziz University, Jeddah, KSA. \\ Email: akokandi@kau.edu.sa \\ Received September $18^{\text {th }}, 2013$; revised October $15^{\text {th }}, 2013$; accepted October $23^{\text {rd }}, 2013$
}

Copyright (C) 2013 Amal A. Kokandi. This is an open access article distributed under the Creative Commons Attribution License, which permits unrestricted use, distribution, and reproduction in any medium, provided the original work is properly cited.

\begin{abstract}
Objectives: Acne is a common skin disease especially in adolescents and young adults. Several pathogenetic factors are implicated in causing the disease. Typically acne flares and remits in its course. Several factors can cause flares. The aim of this study was to assess the perceived factors of acne flares and the idea of sun creams causing skin darkening. Methods: A questionnaire study of the perceived factors in causing acne flares was studied in university female students. These included food, stress, hygiene and menstrual periods. In addition, the effect of sun creams on causing skin darkening was asked. Results: Food was implicated by $43.2 \%$ of patients. Stress and menstrual periods were thought to exacerbate acne by more than $70 \%$ of patients while $80 \%$ of patients did not think bad hygiene worsened acne. $40 \%$ of patients thought the use of sun creams cause skin darkening. Conclusion: More controlled studies are needed to explore the effect of food on acne. Dermatologists need to explain the benefit of using sun creams especially to acne patients while using standard treatments.
\end{abstract}

Keywords: Acne; Precipitating Factors; Food; Sun Creams

\section{Introduction}

Acne is a common skin disease especially in adolescents and young adults [1]. Several pathogenetic factors are implicated in causing the disease. Typically acne flares and remits in its course. Many factors were previously implicated in causing acne and acne flares. These factors include food as precipitating agent, stress, menstrual periods, facial hygiene and marital status [2-4]. Data from our region are scarce.

Most of the patients will be prescribed one of the retinoid creams. These creams are known to make the user photosensitive [5] necessitating the avoidance of sun and the use of sun protecting creams. Some adults are reluctant to use sun creams because of the belief that sun creams cause skin darkening.

In this study perceived factors in causing acne flares were studied. These included food, stress, hygiene and menstrual periods. In addition, to the question about sun creams if they cause skin darkening or not was asked.

\section{Methods}

Subjects: Female students attending King Abdulaziz uni- versity medical administration dermatology clinic (female section) with acne complaints.

Procedure: Verbal informed consent was obtained from all patients and they were assured that their participations were voluntary and their responses were anonymous and confidential.

A medical history was taken in addition to the acnerelated complaint. An assessment of acne was made using the global acne grading system or GAGS [6]. GAGS considers six locations on the face and chest/upper back, with a factor for each location based roughly on surface area, distribution, and density of pilosebaceous units. The severity was graded as mild if the score was 1 - 18, moderate with scores from 19 to 30 , severe with scores from 31 to 38 , and as very severe if the score is more than 38 .

A questionnaire was filled by the investigator by asking the patients about their opinion and to reply by yes or no or do not know. They were asked if they think that food worsens their acne, and if yes which food they think most does, if acne is worsened by bad hygiene, if acne worsened by stress, if acne is affected by the menstrual period, if acne is affected (worsened or improved by marriage) and if using sun protecting creams cause skin 
darkening. This questioned was added because most of acne treatment modalities necessitate the use of sun protection cream and some patients were reluctant to use them. The study protocol was approved by local research ethics committee.

Analysis: The statistical analysis was performed using the SPSS software version 16.0.

\section{Results}

A total of 125 responses were collected. Age of patients ranged from 16 - 39 years (mean 22.2, SD 3.96). Severity ranged from $5-37$ (mean $\pm \mathrm{SD}, 15.54 \pm 5.21)$. Disease duration ranged from $0.3-20$ years (mean $\pm \mathrm{SD}, 5.7 \pm$ 3.18 years). The responses to the questions are summarized in Table 1 and Figure 1.

Acne was implicated to be worsened by certain food by $43.2 \%$ of patients. While $49.6 \%$ of patients did not think food was worsening their acne. The main food implicated in worsening acne was chocolate $(66.7 \%$ of patients agreeing food is causing acne), followed by fatty food and chili (31.5\% and $20.4 \%$ respectively). Less commonly nuts, fizzy drinks and other food items were implicated. A list of food items implicated in worsening acne is presented in Table 2 and Figure 2. Eighty percent of acne patients thought that bad hygiene does not affect or worsen acne. Stress and menstrual period were thought be affecting acne flares by $72.8 \%$ and $78.4 \%$ of patients respectively. Marriage was thought to be affecting acne by $34.4 \%$ of patients, while $28.8 \%$ thought that it does not have a role. Regarding the sunscreen use, $40 \%$ of patients thought that it causes skin darkening and only $27.2 \%$ of patients did not think it causes skin darkening.

\section{Discussion}

Acne is one of the most common complaints in adolescents and early adulthood. Several beliefs still exist regarding the cause and the precipitating factors of acne. In this study some of the factors thought to be related to acne flares were studied in university female students. In addition to the question about the concept of sun creams causing skin darkening was asked.

Food is still debatable issue especially in relation to acne. The earliest studies performed to declare the effect of food, namely, chocolates, milk and roasted nuts were small, uncontrolled and for short periods only $[7,8]$.

Food with high glycemic load are implicated in worsening acne in addition to milk and dairy products $[9,10]$. Soft drinks were considered the most aggravating food item by Pakistani students [11]. On the other hand some food is thought to improve or protect against acne like fish oil [12] and Mediterranean food [13,14]. In this study more than $40 \%$ accused food of worsening acne. Interestingly, chocolate followed by fatty food were the
Table 1. Summary of responses of acne patients to the questions.

\begin{tabular}{|c|c|c|c|}
\hline & Yes (\%) & No (\%) & $\begin{array}{c}\text { Do not } \\
\text { know (\%) }\end{array}$ \\
\hline Is acne worsened by food? & $54(43.2)$ & $62(49.6)$ & $9(7.2)$ \\
\hline $\begin{array}{c}\text { Is acne precipitated by } \\
\text { bad hygiene? }\end{array}$ & $22(17.6)$ & $100(80)$ & $3(2.4)$ \\
\hline Is acne worsened by stress? & $91(72.8)$ & $30(24)$ & $4(3.2)$ \\
\hline Is acne affected by the period? & $98(78.4)$ & $24(19.2)$ & $3(2.4)$ \\
\hline $\begin{array}{l}\text { Does marriage affect acne } \\
\text { in anyway? }\end{array}$ & $43(34.4)$ & $36(28.8)$ & $46(36.8)$ \\
\hline $\begin{array}{l}\text { Does sunscreen use cause } \\
\text { skin tanning? }\end{array}$ & $50(40)$ & $34(27.2)$ & $41(32.8)$ \\
\hline
\end{tabular}

$\%$ the percentage of responses out of total responses (125).

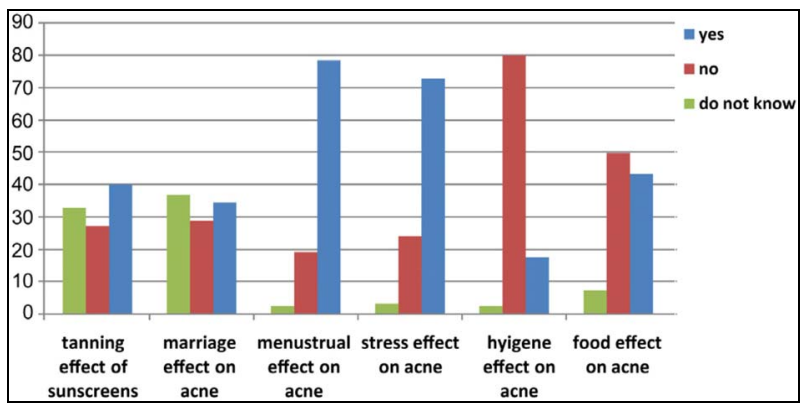

$\%$ the percentage of responses out of total responses (125).

Figure 1. Summary of responses of acne patients to the questions.

Table 2. Types of food implicated in worsening acne.

\begin{tabular}{ccc}
\hline Food & Number of cases & Percentage \\
\hline chocolate & 36 & 66.7 \\
fatty food & 17 & 31.5 \\
chili & 11 & 20.4 \\
nuts & 8 & 14.8 \\
fizzy drinks & 5 & 9.3 \\
chips & 3 & 5.6 \\
sweets & 2 & 3.7 \\
dairy products & 1 & 1.9 \\
aubergiene & 1 & 1.9 \\
eggs & 1 & 1.9 \\
bananas & 1 & 1.9 \\
fish & 1 & 1.9 \\
caffiene & 1 & 1.9 \\
pastries & 1 & 1.9 \\
iron deficiency & 1 & 1.9
\end{tabular}

The percentage is the percentage implicating the food out of total cases implicating food as a causative agent for acne flare (54). 


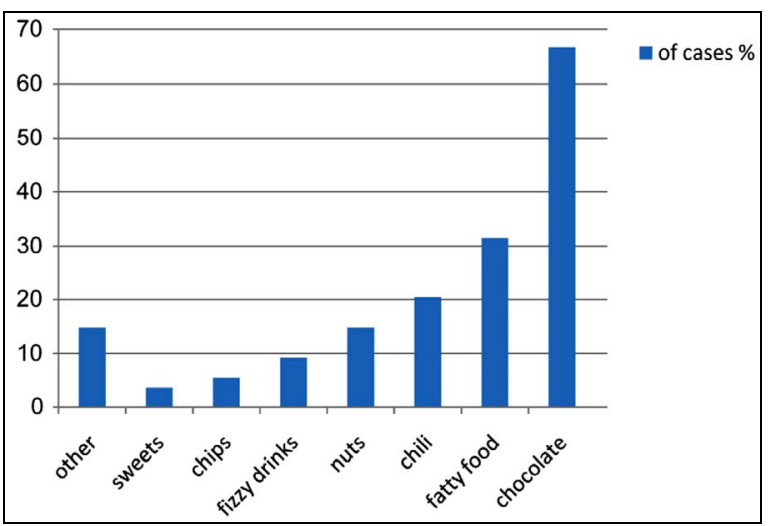

The percentage is the percentage implicating the food out of total cases implicating food as a causative agent for acne flare (54).

Figure 2. Food items implicated in worsening acne.

most culprits. There is still a need for more controlled studies regarding the effect of food on acne.

Sometimes the stigma of cleanlessness is feared by some acne patients $[2,4]$. In some societies still poor hygiene is thought to be a cause of acne [15], or acne to be relieved by frequent face washing [11]. In this study $80 \%$ of patients thought that bad hygiene does not worsen acne but still $17.6 \%$ of patients thought bad hygiene is a factor. Acne is known to cause a significant psychological burden on patients. The effect of feeling guilty about worsening it by bad hygiene may cause an added negative effect on patients.

Stress was reported to be aggravating factor of acne $[15,16]$. In this study more than $70 \%$ of patients thought acne was worsened by stress. Similar results were reported by previous studies in different societies $[17,18]$. Stress seems to be a factor in our society too.

Acne is known to be associated with the polycystic ovarian syndrome and affecting their quality of life [18]. Period irregularity is one of PCO manifestations and higher testosterone level than normal subjects are manifestations too. More than $78 \%$ of patients in this study claimed that acne flared in relation to the menstrual period. This might be explained by hormonal variation throughout the menstrual cycle. Only $34.4 \%$ of patients thought that acne is related to the marital state. Apparently there is not a link between marital state or sexual activity, and acne flares.

Most acne patients will be prescribed topical or systemic treatment that will cause them photosensitive. This necessitates the use of sun protecting creams. There is a misconception of sun creams causing darkening of skin. This concept might came from the fact that some sun cream users are feeling sun safe and exposing themselves to the sun for longer times. The other explanation might be that some sun cream users are not applying the creams in enough amounts or not renewing the application as needed. In this study $40 \%$ of patients thought that sun protecting creams cause skin darkening and only $27.2 \%$ think that it does not. This might warrant dermatologist to explain the benefit of using sun protecting creams to avoid sun burning or unwanted facial tanning especially for patients on treatments with photosensitizing agents. The counseling should be individualized [19].

\section{Conclusion}

More than $40 \%$ of acne patients think that food is a causative factor and more than $70 \%$ of patients think that stress and menstrual period is an exacerbating factor. More research is needed in regard to food as causative or precipitating acne lesions. Counseling regarding the use of sun protecting creams should be part of the clinical management of acne patients especially if they will be on treatments specially retinoids.

\section{REFERENCES}

[1] B. Razany and C. Kahl, "Epidemiologie der Acne Vulgaris," Journal of the German Society of Dermatology, Vol. 4, No. 1, 2006, pp. 8-9.

[2] I. Brajac, L. Bilic-Zulle, M. Tkalcic, K. Loncarek and F. Gruber, "Acne Vulgaris: Myths and Misconceptions among Patients and Family Physicians," Patient Education and Counseling, Vol. 54, No. 1, 2004, pp. 21-25. http://dx.doi.org/10.1016/S0738-3991(03)00168-X

[3] J. E. Rasmussen and S. B. Smith, "Patient Concepts and Misconceptions about Acne," Archives of Dermatology, Vol. 119, No. 7, 1983, pp. 570-572. http://dx.doi.org/10.1001/archderm.1983.01650310032006

[4] J. K. Tan, K. Vasey and K. Y. Fung, "Beliefs and Perceptions of Patients with Acne," Journal of American Academy of Dermatology, Vol. 44, No. 3, 2001, pp. 439-445. http://dx.doi.org/10.1067/mjd.2001.111340

[5] S. V. Tripathi, C. J. Gustafson, K. E. Huang and S. R. Feldman," Side Effects of Common Acne Treatments," Expert Opinion in Drug Safety, Vol. 12, No. 1, 2013, pp. 39-51. http://dx.doi.org/10.1517/14740338.2013.740456

[6] A. Doshi, A. Zaheer and M. J. Stiller, "A Comparison of Current Acne Grading Systems and Proposal of a Novel System," International Journal of Dermatology, Vol. 36, No. 6, 1997, pp. 416-418. http://dx.doi.org/10.1046/j.1365-4362.1997.00099.x

[7] J. D. Grant and P. C. Anderson, "Chocolate and Acne: A Dissenting View," Missouri Medicine, Vol. 62, 1965, pp. 459-460.

[8] P. C. Anderson, "Foods as the Cause of Acne," American Family Physician, Vol. 3, No. 3, 1971, pp. 102-103.

[9] N. H. Ismail, Z. A. Manaf and N. Z. Azizan, "High Glycemic Load Diet, Milk and Ice Cream Consumption Are Related to Acne Vulgaris in Malaysian Young Adults: A Case Control Study," BMC Dermatology, Vol. 12, 2012, p. 13. http://dx.doi.org/10.1186/1471-5945-12-13

[10] J. Burris, W. Rietkerk and K. Woolf, "Acne: The Role of Medical Nutrition Therapy," Journal of the Academy of 
Nutrition and Dietetics, Vol. 113, No. 3, 2013, pp. 416430. http://dx.doi.org/10.1016/j.jand.2012.11.016

[11] G. Ali, K. Mehtab, Z. A. Sheikh, H. G. Ali, S. Abdel Kader, H. Mansoor, S. Altaf, S. Qamar and S. S. Khwaja, "Beliefs and Perceptions of Acne among a Sample of Students from Sindh Medical College, Karachi," The Journal of the Pakistan Medical Association, Vol. 60, No. 1, 2010, pp. 51-54.

[12] G. Khayef, J. Young, B. Burns-Whitmore and T. Spalding, "Effects of Fish Oil Supplementation on Inflammatory Acne," Lipids in Health and Disease, Vol. 11, 2012, p. 165. http://dx.doi.org/10.1186/1476-511X-11-165

[13] S. Rezaković, Z. B. Mokos and A. Basta-Juzbašić, "Acne and Diet: Facts and Controversies," Acta dermatovenerologica Croatica, Vol. 20 No. 3, 2012, pp. 170- 174.

[14] N. Skroza, E. Tolino, L. Semyonov, I. ProiettiI, N. Bernardini, F. Ncolucci, G. La Viola, G. Del Prete, R. Saulle, C. Potenza and G. La Torre, "Mediterranean Diet and Familial Dysmetabolism as Factors Influencing the Development of Acne," Scandinavian Journal of Public Health, Vol. 40, No. 5, 2012, pp. 466-474. http://dx.doi.org/10.1177/1403494812454235

[15] C. E. Cheng, B. Irwin, D. Mauriello, L. Liang, A. Pappert and A. B. Kimball, "Self-Reported Acne Severity, Treatment, and Belief Patterns across Multiple Racial and Eth- nic Groups in Adolescent Students," Pediatric Dermatology, Vol. 27, No. 5, 2010, pp. 46-452. http://dx.doi.org/10.1111/j.1525-1470.2010.01286.x

[16] A. A. Al Robaee, "Prevalence, Knowledge, Beliefs and Psychosocial Impact of Acne in University Students in Central Saudi Arabia," Saudi Medical Journal, Vol. 26, No. 12, 2005, pp. 1958-1961.

[17] J. Green and R. D. Sinclair, "Perceptions of Acne Vulgaris in Final Year Medical Student Written Examination Answers," Australasian Journal of Dermatology, Vol. 42, No. 2, 2001, pp. 98-101. http://dx.doi.org/10.1046/j.1440-0960.2001.00489.x

[18] S. Elsenbruch, S. Hahn, D. Kowalsky, A. H. Offner, M. Schedlowski, K. Mann and O. E. Janssen, "Quality of Life, Psychosocial Wellbeing, and Sexual Satisfaction in Women with Polycystic Ovary Syndrome," Journal of Clinical Endocrinology and Metabolism, Vol. 88, No. 12, 2003, pp. 5801-5807. http://dx.doi.org/10.1210/jc.2003-030562

[19] P. Magin, D. Pond, W. Smith and A. Watson, "A Systematic Review of the Evidence for 'Myths and Misconceptions' in Acne Management: Diet, Face-Washing and Sunlight," Family Practice, Vol. 22, No. 1, 2005, pp. 6270. http://dx.doi.org/10.1093/fampra/cmh715 